\title{
Treatment selections of 239 patients with blepharospasm and Meige syndrome over 11 years
}

Department of Ophthalmology, UMDNJ, New Jersey Medical School, Newark, USA

J A Mauriello, Jr S Dhillon

T Leone

B Pakeman

R Mostafavi

M C Yepez

Correspondence to: J A Mauriello, Jr, MD, Department of

Ophthalmology, UMDNJ, New Jersey Medical School, Doctors Office Center, 90 Bergen Street, Newark, NJ 07103, USA.

Accepted for publication 13 September 1996

Joseph A Mauriello, Jr, Shamina Dhillon, Tina Leone, Basil Pakeman, Ramin Mostafavi, Maria C Yepez

\begin{abstract}
Background-A retrospective review of 239 patients with benign essential blepharospasm and Meige syndrome was performed in order to determine patients' long term treatment preferences.

Methods-Of 239 patients evaluated, 228 received local injections of botulinum toxin, type $A$, into the eyelid and facial musculature over 11 years.

Results-Of 228 patients, 202 (72.1\%) were still treated with botulinum toxin, type $A$. Eighteen patients $(6.9 \%)$ no longer received botulinum toxin injections and sought no other treatment. Five patients (2.2\%) had apparent remission of their disease after injection. Three patients (1.3\%) ultimately obtained relief from orbicularis muscle extirpative surgery and required no additional treatment. Two of the 11 patients $(4.6 \%)$ who chose not to receive botulinum toxin injections were successfully treated with other modalities: psychotherapy (one patient) and oral haloperidol (one patient).

Conclusion-While botulinum toxin is the most highly effective treatment for benign essential blepharospasm and Meige syndrome over a long period of time, adjunctive oral drug therapy, including minor tranquillisers as well as eyelid surgery, may augment its effectiveness.

(Br $\mathcal{F}$ Ophthalmol 1996;80:1073-1076)
\end{abstract}

Essential blepharospasm is an idiopathic focal dystonia that consists of involuntary closure of the eyelids and eyebrow region that may render the inflicted patients functionally blind. ${ }^{1-5}$ It usually starts with increased frequency of blinking and may progress to involuntary prolonged muscular contractions.

Oromandibular dystonia or Meige syndrome consists of involuntary contractures of the muscles around the mouth innervated by the other nerves especially the fifth, tenth, and twelfth cranial nerves in association with blepharospasm. Meige syndrome was described by Andre Meige, the French neurologist in 1910. Eyelid involvement may predate or follow midfacial, oral, mandibular, laryngeal, or pharyngeal involvement. Associated involuntary movements include facial grimacing, frowning, head titubation, torticollis, and spastic dysphonia. Essential blepharospasm, Meige syndrome, and spasmodic torticollis are dystonic movement disorders or dyskinesias that typically present in the fifth and sixth decades of life. ${ }^{6-10}$

Botulinum toxin, type A, blocks the release of the neurotransmitter acetylcholine at the muscle end plate and is currently the treatment of choice for benign essential blepharospasm and Meige syndrome.

We previously reviewed 50 patients initially treated with botulinum toxin injections between September 1983 and June 1984 and followed for 7 years. The study included 34 patients with blepharospasm, 13 with hemifacial spasm, and four with Meige syndrome. ${ }^{10}$ Of the original 50 patients, $26(52 \%)$ continued to return for periodic injections and included 18 of 33 patients with blepharospasm, seven of 13 with hemifacial spasm, and one of four with Meige syndrome. ${ }^{10}$

A long term study of a large cohort of patients with benign essential blepharospasm and Meige syndrome and their treatment selections has not been reported. ${ }^{1-10} \mathrm{We}$, therefore, analysed 239 patients who were examined and treated for 11 years in order to determine: (1) whether botulinum toxin injections are accepted by patients on a long term basis, and (2) the role of other treatment modalities including oral medications and surgery in controlling facial contractures.

\section{Materials and methods}

Charts of all patients with a diagnosis of benign essential blepharospasm and Meige syndrome (with eyelid involvement) were reviewed from the oculoplastics division of the Department of Ophthalmology of UMD-New Jersey Medical School, Newark, New Jersey, and from the private practice of one of us (JAM) from October 1983 to October 1994.

Botulinum toxin (Botox, Allergan Pharmaceutical, USA), 12.5 units ( $5 \mathrm{ng}$ ) diluted in 4 $\mathrm{ml}$ of non-preserved saline, was distributed in the pretarsal orbicularis muscle of the upper eyelid medially and laterally (2.5 units or 0.1 $\mathrm{ml}$ in each location) and similarly in the lower eyelids ( 2.5 units or $0.1 \mathrm{ml}$ in each location) and the lateral canthus on the affected side (2.5 units) at the first treatment session. (It should be noted that there are two preparations of Botox available in the UK that are of different potencies.) The injections were performed bilaterally with a tuberculin syringe and a 30 gauge needle. Since the orbicularis muscle is located just below the skin with no intervening subcutaneous fat, there is no need for electromyographic guidance. 
Midfacial and mandibular spasms were treated with a total of 5 units injected in two separate locations in the upper face in the area of the malar eminence and just medial to the mid aspect of the inferior orbital rim. In patients with spasms of the jaw, 5 units were given at the angle of the jaw in the muscle just above the condyle of the jaw (below the zygomatic arch) but not into the joint space. Doses of 5-7.5 units were given in two to three sites along the ramus of the jaw. Injections adjacent to the corner of the mouth were avoided because of the possibility of induced drooling, asymmetric smile, or inadvertent biting of a flaccid buccal mucosa.

Two weeks after injection, the effects of the treatment were assessed on subjective and objective criteria. The percentage of 'overall improvement' was subjectively determined by each patient and compared with pretreatment. The relative weakness of the eyelids on forced closure was graded objectively by the examiner on a scale from +1 to $+4(+1$ is the least amount of force generated). Evidence of residual spasm was also evaluated by the examiner. Patients with a $75-80 \%$ improvement who were subjectively 'satisfied' with improvement in performing daily functions and who had minimal objective evidence of residual spasm were asked to return only when the spasm recurred to almost pretreatment intensity.

Patients not satisfied with their result and with residual eyebrow and/or upper eyelid spasms received injections above the medial eyebrows ( 5 units in two sites above the medial aspect of the brow). Additional drug was given in the upper lid (5 units in each lid) unless ptosis or superior rectus weakness was present. Patients with residual facial contractures were re-examined in 2 weeks ( 4 weeks after the initial injection). At this time, eyelid injections ( 5 units additionally in each upper lid) were repeated at 2 week intervals until a therapeutic effect was achieved. The cumulative dose of the previous week's injections was given on re-injection several months later after the successful dose was established. For example, if repeat upper eyelid injections were necessary 2 weeks after the initial injection, a total of 10 units (rather than 5 units) was given in each upper eyelid on re-injection (since 5 units were given in each eyelid on the first injection and 5 units were injected 2 weeks later).

In general, patients were asked to return for another treatment session when the effects of the drug were sufficiently dissipated that involuntary eyelid and facial spasms were almost at the pretreatment level. The following data were obtained from the chart review: diagnosis, duration of disease before treatment with botulinum toxin, type $A$, median number of injections and median duration of effect, and other treatments including oral pharmacological agents and surgery, and complications. Patients no longer followed were interviewed by telephone.

All patients with a satisfactory response to botulinum toxin, who were previously prescribed oral pharmacological agents, were asked to consult the prescribing physician in order to stop such medications. In addition, patients with an unsatisfactory response to injections were asked to consult their primary care physician to consider administration of an oral agent to possibly enhance or replace the botulinum treatment.

\section{Results}

In all, 239 patients included in the study were examined or treated with botulinum toxin, type A, injections over an 11 year period. A total of 222 patients had benign essential blepharospasm (84 males and 138 females) and 17 patients had Meige syndrome (four males and 13 females); 228 patients were treated with botulinum toxin.

The mean duration of action was 14.9 weeks for 211 patients with blepharospasm and 11 weeks for the 17 patients with Meige syndrome. Not all patients were followed for 11 years. The patients received the injections at expected timely intervals and there was no evidence of tolerance to the drug. Forty five of $228(19.7 \%)$ patients required brow injections and 17 of $228(7.5 \%)$ facial injections. It was not necessary to increase the dose of drug injected above the eyebrow, in the lower eyelid, and in the lateral canthus.

All side effects were transient and included pain on injection. Incomplete blink and decreased lower eyelid 'lacrimal pump' caused the following signs and symptoms: burning, itching, tearing, foreign body sensation, conjunctival injection, photophobia, blurred vision, and, rarely, recurrent corneal erosion $(36 \%)$. These symptoms decreased in intensity 2-4 weeks after treatment. Mild, transient ptosis for 2-4 weeks occurred in $14 \%$ of patients. Lower lid ectropion and entropion each occurred in one patient $(2 \%)$; both occurred in patients with pre-existing lower eyelid laxity.

\section{Still treated}

A total of 94 patients continued treatment with the botulinum toxin injections. Of these, 89 were diagnosed with blepharospasm and five with Meige syndrome (Table 1). Patients with blepharospasm received a median number of 12 injections over a median treatment period of 52 months. Patients with Meige syndrome, all with significant eyelid involvement, had a median number of five injections over a median period of 35 months for each patient. The greatest number of treatments received by one patient was 42 and the longest duration of treatment was 122 months.

\section{Still treated elsewhere}

Eighteen patients continued to obtain injections at a location closer to their home than our institution.

\section{Treated until death}

Six patients with benign essential blepharospasm were treated with botulinum toxin until death (six injections over 35 months) and one patient with Meige syndrome received 22 injections over a 65 month period. 
Table 1 Treatment selections of 239 patients with blepharospasm and Meige syndrome evaluated for and/or treated with botulinum toxin over 11 years

\begin{tabular}{lc}
\hline Treatment selections & Number of patients \\
\hline Botulinum toxin as primary treatment & $202(84.5 \%)$ \\
Still treated with botulinum toxin & $94(39.3 \%)$ \\
Still treated elsewhere with botulinum toxin & $18(7.5 \%)$ \\
Treated with botulinum toxin until death & $7(2.9 \%)$ \\
Treated with botulinum toxin until lost to follow up & $83(34.7 \%)$ \\
No longer treated with botulinum toxin because of transport difficulties & $4(1.7 \%)$ \\
Chose to cope with disease without further treatment after botulinum & $6(2.5 \%)$ \\
toxin injection & \\
Treatment with botulinum toxin no longer required owing to resolution of & $5(2.5 \%)$ \\
condition & $8(3.3 \%)$ \\
Inadequate response to botulinum toxin & $3(1.3 \%)$ \\
Radical orbicularis myectomy eyelid surgery after botulinum toxin & $11^{\star}(4.6 \%)$ \\
Never treated with botulinum toxin & \\
\hline
\end{tabular}

^See Table 2 .

Table 2 Current status of 11 patients never treated with botulinum toxin

\begin{tabular}{ll}
\hline Current treatment status & Number of patients \\
\hline Psychotherapy alone & 1 \\
$\begin{array}{l}\text { Drug therapy only } \\
\text { No further treatment (disease }\end{array}$ & 1 \\
$\quad$ unchanged) & 4 \\
$\begin{array}{l}\text { Disease somewhat improved without } \\
\quad \text { further treatment }\end{array}$ & 1 \\
$\begin{array}{l}\text { Died without any additional treatment } \\
\text { Lost to follow up }\end{array}$ & 1 \\
\hline
\end{tabular}

Treated with botulinum toxin until lost to follow up Eighty three patients who were treated with botulinum toxin were lost to follow up over the 11 year period. This group of patients had a median number of three injections over a median treatment period of 8 months per patient.

No longer treated because of transport difficulties Four patients had a fairly good response to botulinum toxin injections but owing to difficulty in obtaining transportation for medical treatment sought no further treatment of any type.

\section{Chose to cope with disease without further} treatment after botulinum toxin injection

Six patients chose to cope with their disease rather than receive additional treatment. Two such patients were influenced by the pain on injection and temporary blepharoptosis, respectively.

\section{Treatment no longer required owing to resolution of condition}

Five patients with benign essential blepharospasm reported unexpected remission and required no further treatment.

\section{Inadequate response to botulinum toxin}

Eight patients felt the injections did not last long enough and opted for no further injections.

\section{Radical orbicularis myectomy eyelid surgery after botulinum toxin}

Three patients treated with botulinum toxin sought a more permanent solution to their problem. The three patients with benign essential blepharospasm who underwent muscle extirpative surgery required no additional botulinum toxin treatment.
Patients never treated

Eleven patients with blepharospasm were seen in consultation but for a variety of reasons were never treated with botulinum toxin and chose other treatment methods (Table 2).

One patient sought relief from psychotherapy and enjoyed significant improvement in the blepharospasm for more than 3 years after cessation of all treatment. Another patient was treated for 4 years with oral benzhexol.

SURGERY AS ADJUNCTIVE TREATMENT TO BOTULINUM TOXIN INJECTIONS

Seven patients had blepharoplasty surgery which was combined with levator aponeurotic reinsertion (two patients) and limited upper eyelid orbicularis myectomy (two patients). These additional seven patients continued to receive botulinum toxin injections at expected intervals with no increased duration per injection but with a subjective improvement compared with presurgical effectiveness of the injections.

ORAL PHARMACOLOGICAL AGENTS AS ADJUNCTIVE TREATMENT TO BOTULINUM TOXIN INJECTIONS

In addition to botulinum toxin, 99 patients opted for oral pharmacological treatment from their family physician or neurologist (Table 3 ). The ophthalmologist (JAM) did not prescribe any medication. The most common classifications of drugs prescribed were minor tranquillisers (51 patients) including alprazolam (Xanax) (25 patients), diazepam (Valium) (16 patients), and lorazepam (Ativan) (10 patients). Other drugs used included the muscle relaxant, baclofen (Lioresal) (16 patients), anticholinergic medications, benzhexol (trihexyphenidyl, Artane) (14 patients), and antiseizure medications, clonazepam (Clonopin) (12 patients) and carbamazepine (Tegretol) (six patients). Five patients received multiple drugs: diazepam and benzhexol (two patients) and benzhexol and baclofen combined with ethopropazine (Parsidole) (one patient), alprazolam (one patient), and Parsidole and haloperidol (Haldol) (one patient).

\section{Discussion}

Based on this retrospective study of 239 patients with idiopathic blepharospasm and Meige syndrome over an 11 year period, botulinum toxin injections proved to be an effective treatment. In all, 228 of 239 initially examined

Table 3 Drug therapy combined with botulinum toxin Table 3 Drug therapy com
injections in 99 patients

\begin{tabular}{ll}
\hline Type of drug & Number of patients \\
\hline Minor tranquillisers & $51(51.5 \%)$ \\
alprazolam (Xanax) & $25(25.3 \%)$ \\
diazepam (Valium) & $16(16.2 \%)$ \\
lorazepam (Ativan) & $10(10.1 \%)$ \\
$\begin{array}{c}\text { Muscle relaxant } \\
\text { baclofen (Lioresal) }\end{array}$ & $16(16.2 \%)$ \\
$\begin{array}{c}\text { Anticholinergic } \\
\text { benzhexol (Artane) }\end{array}$ & $14(14.1 \%)$ \\
Antiseizure & $18(18.2 \%)$ \\
clonazepam (Clonopin) & $12(12.1 \%)$ \\
carbamazepine (Tegretol) & $6(6.1 \%)$ \\
\hline
\end{tabular}

^Five patients received multiple drugs: diazepam and benzhexol (two patients); benzhexol and baclofen combined with ethopropazine (one patient); alprazolam (one patient); ethopropazine and haloperidol (one patient). 
were treated with botulinum toxin. Follow up showed that of 228 patients treated, 202 patients $(72.1 \%)$ were still being treated with botulinum toxin, type $\mathrm{A}$.

In this study, the mean duration of action was 14.9 weeks for 211 patients with blepharospasm and 11 weeks for 17 patients with Meige syndrome. Only eight patients had an inadequate response to the botulinum toxin injections while six patients chose to cope with disease without further treatment after botulinum toxin injection. Tolerance to the drug was not a problem in this series. ${ }^{112}$ Antibodies have not been found in a small number of patients treated for blepharospasm. ${ }^{12}$ Theoretically, toxin that is incorrectly formulated, dried, or rehydrated may form inactive, antigenic toxin (toxoid).

The fact that five patients had spontaneous resolution of the blepharospasm after botulinum injections is of interest. While the reason for the resolution is unclear, physicians should probably counsel their patients with benign essential blepharospasm and Meige syndrome that symptoms may rarely abate after treatment with botulinum toxin. It is impossible to ascertain whether the botulinum toxin itself influenced the natural history of the facial dyskinesias.

Other treatments, to the exclusion of botulinum toxin injections, included radical orbicularis myectomy surgery (three patients), psychotherapy (one patient), and oral anticholinergic medication (one patient). Such modalities were ultimately chosen as the primary treatment by a small minority of the 239 patients in this series.

Adjunctive treatments to the botulinum toxin injections included eyelid surgery and oral anxiolytic medications. Six patients underwent excision of excess skin and plication of the levator aponeurosis. Drug therapy was prescribed by the primary care physician or neurologists for 99 patients. Most patients obtained additional relief from anxiolytic medications (51 patients). These data strongly suggest that while botulinum toxin helps to control the symptoms of blepharospasm and Meige syndrome, limited myectomy and pharmacological agents may enhance the effectiveness of the injections. Since antianxiety medications are the most common drugs taken along with botulinum, control of stress by any technique may similarly augment the effects of botulinum toxin.

We conclude that botulinum A toxin is a well tolerated long term treatment of blepharospasm. The greatest management problem lies in patients who do not respond to the drug.

This work was supported in part by an unrestricted Departmenthal grant from Research to Prevent Blindness, Inc, NY, and from the Lions Eye Research Foundation, Newark, NJ 07103, USA.

1 Scott AN, Kennedy RA, Stubbs HA. Botulinum A toxin injections as a treatment for blepharospasm. Arch Ophthal mol 1985;103:347-50.

2 Kraft SP, Long AE. Botulinum toxin injections in the treatment of blepharospasm, hemifacial spasm, and eyelid ment of blepharospasm, hemifacial spasm, and

3 Frueh BR, Musch DC. Treatment of facial spasm with Frueh BR, Musch DC. Treatment of facial spasm with
botulinum toxin: an interim report. Ophthalmology 1986 93:917-23.

4 Shorr N, Seiff RS, Kopelman J. The use of botulinum toxin in blepharospasm. Am $\mathcal{F}$ Ophthalmol 1985;99:917-23.

5 Cohen DA, Savino PJ, Stern MB, Hurtig HI. Botulinum injection therapy for blepharospasm: a review and report of 75 patients. Clin Neuropharmacol 1986;9:415-29.

6 Elston JS. Long-term results of treatment of idiopathic blepharospasm with botulinum toxin injections. $\mathrm{Br} f \mathrm{Oph}$ thalmol 1987;71:664-8.

7 Dutton J, Buckley EG. Long-term results and complicaDutton J, Buckley EG. Long-term results and complicaspasm. Ophthalmology 1988;95:1529-34.21.

8 Mauriello JA. Blepharospasm, Meige syndrome, and hemifacial spasm: treatment with botulinum toxin. Neurology 1985;35:1499-500

9 Mauriello JA, Coniaris H, Haupt EJ. Use of botulinum toxin in the treatment of one hundred patients with facial dyskinesias. Ophthalmology 1987;94:976-9.

10 Mauriello JA, Aljian J. The natural history of treatment of facial dyskinesias with botulinum toxin: a study of fifty consecutive patients over seven years. $\mathrm{Br} \mathcal{f}$ Ophthalmol 1992;76:349-52.

11 Gonnering RS. Negative antibody response to long term treatment of facial spasm with botulinum toxin. Am $f O p h-$ treatment of facial spas

12 Borodic GE, Pearce LB, Johnson E. Clinical and scientific aspects of botulinum A toxin. Ophthalmol Clin N Am 1991; 4:491-503. 